\title{
EFFECT ON AODV ROUTING PROTOCOL UNDER BLACKHOLE ATTACK IN VANET
}

\author{
Dr. Ajay N. Upadhyaya \\ Computer Engineering Department, \\ Ahmedabad Institute of Technology, Ahmedabad, India \\ Dr. J.S. Shah \\ Computer Engineering Department, Ex. Principal, \\ Government Engineering College, Patan, India
}

\begin{abstract}
Vehicular Ad-hoc Networks (VANETs) is an advancement over Mobile Ad Hoc Networks (MANETS) which is mainly design for inter vehicle communication. VANET is a scalable and unbounded network which is completely independent from the number of nodes. It can be implemented for one or several cities even for whole country. VANET is a self organizing network emergent technology with a promising advantages but having high challenges in its security. VANET communication must be secure and having guarantee that transmitted message is not inserted or modified by any attackers. VANETs is having different security requirement for governing proper vehicular communication. For secure VANET communication first systems have to discover who are the attackers, their capacity and nature to spoil the network communication. In this paper we have done the analysis of different Routing protocols OLSR, DSDV, AODV and DSR in VANET. We selected AODV as a most vulnerable protocol in VANET and presented effect of Blackhole Attack using AODV Protocol with the detail comparative analysis of Packet Drop Rate, Throughput and End to End Delay Parameters.
\end{abstract}

Key words: Security, AODV, Routing Protocol, Blackhole Attack, Routing Attack, VANET.

Cite this Article: Dr. Ajay N. Upadhyaya, Dr. J.S. Shah, Effect on AODV Routing Protocol Under Blackhole Attack in VANET, International Journal of Computer Engineering and Technology 10(3), 2019, pp. 166-174.

http://iaeme.com/Home/issue/IJCET?Volume=10\&Issue $=3$

\section{INTRODUCTION}

Due to recent advancements in vehicular technologies, the need is emerged for vehicle communication. There is a need to provide intelligence to vehicle which is capable to do communication with other vehicles as well as with road side infrastructure. By providing the traffic information to vehicles, vehicle can select the optimal path which will be very helpful 
in big cities having serious traffic problems. If driver aware about traffic conditions on the road ahead will give ability to select optimal path through which time, energy and fuel will be saved. Also communication with the infrastructure will allow vehicle to access different requirement based services. Lots of research work is done by different researcher for VANET under the roof of adhoc network. Security is often considered to be the major "roadblock" in VANET technology. Without effective security VANET becomes useless.

Security Management is main concern for governing different applications of VANET. VANET communication must be secure from different types of attackers. If an attacker changes the contents of data, create unnecessary delay, changing self identity or misbehave in the network it becomes crucial for VANET network. Adoption and growth of VANET is suffer due to the open environment, lack of centralized monitoring and security standards, High mobility of vehicles. This research focuses on different security loopholes in adhoc network specifically in VANET and based on that develop and implement new secure approaches which can give better security for routing attacks specifically in Black Hole Attack.

\section{RELETED WORK}

In [1], author discussed and proposed routing protocol for city area of VANET. By using the Geographical forwarding in city traffic layout they try to improve the routing process. They proposed two different approaches of routing protocol: Proactive and Reactive with the detailing of their working mechanism. Evaluation of protocols is presented using simulator NS 2.30 for different city scenario. Result is analyzed based on different parameters like Average Delay, Average delivery ratio, Average Length of path and Network Overhead. In [2], author discussed about different challenges of routing protocol designing in VANETs by showing the study of different Routing protocols. They presented the comparison of different routing protocols. They classified and discussed the protocols into five categories: Ad hoc Routing Protocols, Position based Routing Protocol, Cluster based Routing Protocol, Broadcasting Protocol, and Geo-casting routing Protocol. Paper is presenting a survey of different routing protocols. In [3], authors discussed about the various vulnerabilities in adhoc network and also discuss different attacks and security mechanisms for mobile ad hoc networks. In paper author mainly discussed about two types of security mechanism: Intrusion detection techniques and different techniques for Secure Routing. Also discussed about three types of intrusion detection: Misuse-based, Anomaly-based and Specification-base. Watchdog \& Pathrater and localized Self-healing Communities were discussed for secure routing techniques In [4], author highlighted and reviewed different security related aspects of VANET.Author have analyzed and said that need to adopt high level of security for secure VANET Transaction. In [5], author done the survey for the field of security in VANET. They discussed different security proposal proposed by various researches. Survey is presented with details analysis of different methods. In [6], author discussed security challenges and problem of VANET with the set of possible solutions. They discussed about different types of attack in VANET and also discussed various proposed solutions. They proposed methods for managing the certificate of normal messages and sending encrypted emergency messages.

In [7], author discussed about AODV and OLSR Routing Protocol with different mobility model by considering different vehicle density parameter. They mainly focused on adaptability of reactive and proactive routing protocol and presented the improvement in result with the simulation in OMNET++. In [8], author discussed about the proactive and reactive routing protocols. They evaluated the performance of AODV and OLSR under different traffic scenario using the network simulator NS-3. With the different metrics like throughput, packet delivery ratio (PDR), and end to end delay they discussed performance of 
AODV and OLSR. In [9], author discussed about important issues for desirable VANET applications with different routing protocols. They presented comparative study of different protocols by considering their forwarding method, scenario, infrastructure and traffic flow information. They also discussed advantages and disadvantages of different protocols with the simulation result by considering parameter of packet delivery ratio and throughput. In [10] paper, author discussed different VANET routing protocols and based on it proposed protocol model having improved functionality. They discussed AODV in details and presented proposed approach with the comparative analysis of by considering parameter like Packet Loss Ratio, Packet Delivery Ratio , Average End-to-End Delay and Normalized Routing Load.

\section{VANET ROUTING PROTOCOL}

To improve the efficiency of routing protocol many routing protocols have been proposed. The high mobility of vehicle creates the more challenging environment for designing efficient routing protocol. Routing protocol is categories in two categories: Topology based routing protocols and Position based routing protocols.

Link information is used for transmitting data packets in Topology based routing. Topology based routing can further categories into two sub categories :Proactive Routing (Table Driven Routing) and Reactive Routing (On Demand Routing).

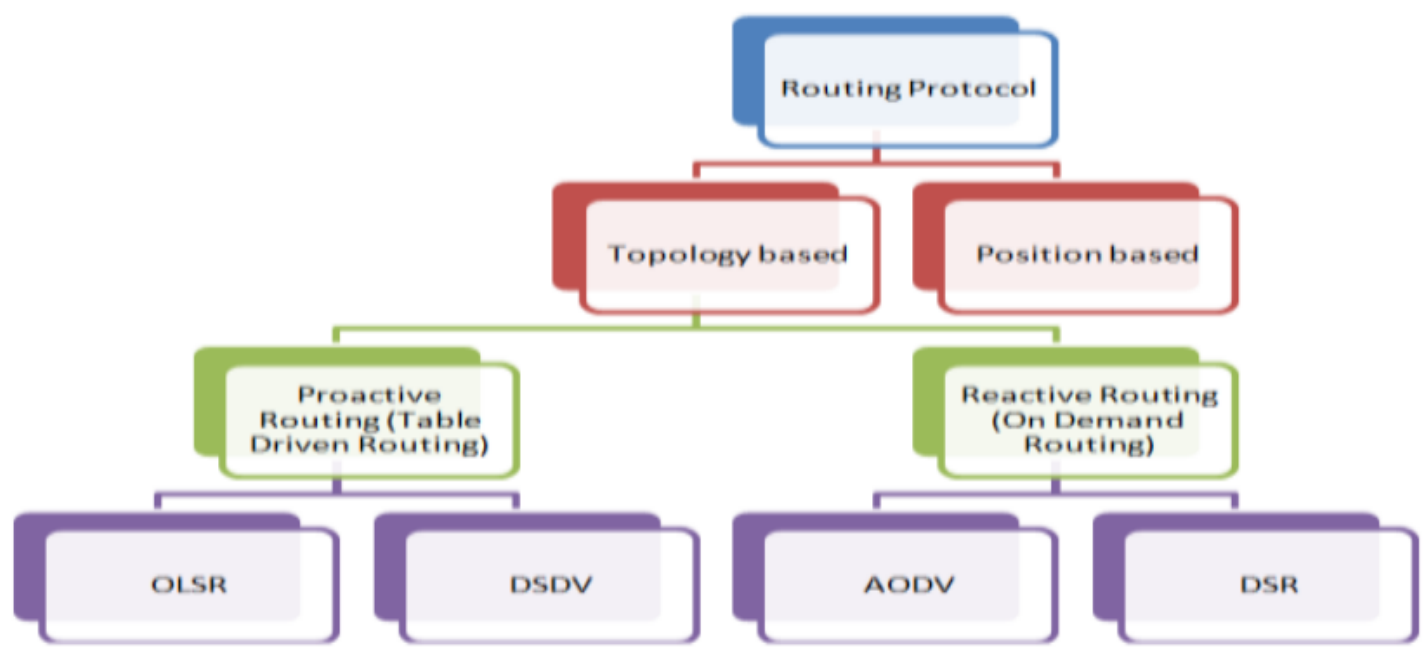

Figure 1 VANET Routing Protocol

A shortest path algorithm is used in Proactive routing protocols which is managing information of all connected nodes. This collected information is further shared with their neighbor. If any changes occur then every node will updates its routing table. This category of protocol is having advantages like they not required Route Discovery and also having low latency which is very useful in real time applications. Also having disadvantages like poor performance in small adhoc network, Memory overhead and Processing Overhead is increase with the expansion of network.

Reactive routing protocol only discovers route towards other node when there is a need of communication is occur. Due to such approach it reduces network traffic. This category of protocol is having advantages like it is not require periodic flooding inside the network and required less bandwidth. Also having disadvantages like latency is high. 
Here briefly discuss two proactive routing protocol: OLSR(Optimized Link State Routing) and DSDV(Destination Sequence Distance Vector) and two reactive routing protocol: AODV(Ad hoc on Demand Distance Vector) and DSR(Dynamic Source Routing).

OLSR: It is a proactive routing protocol which updates its routing Tables periodically. It is using Link state algorithm for managing the route and periodically updates link information with each other. OLSR uses MPR (multipoint relay) which is helpful in reducing the size of control messages and also reduce the number of rebroadcasting nodes. To find the optimal path high bandwidth and processing power is required compare to other protocols.

DSDV: It is a proactive routing protocol, which periodically updates routing table after getting every updates. Each node maintain the details of other node which are available in the network. After notification about any changes, node will notify other nodes in the network through broadcasting.

AODV: AODV is a demand based reactive routing protocol. It only establish the route when any need occurs. It is having route request-response mechanism through which it send the request for finding the route and based on received response it establish the optimal path. It only share the updates to the neighbor using unicast or multicast instead of broadcasting to all. Based on fresh entry of destination sequence number it will establish the best route. It is more preferable for high Packet Delivery Ratio and less Normalized Routing Load but also getting higher Avg. End-to-End delay and Dropped packets compare to other protocols.

DSR: It is demand based reactive routing protocol. It manages the whole route from source to the destination. It also manages the active routes. It manage the route in two phase: Route discovery and Route maintenance. It has lower End to End delay compare to other protocols but more suitable for smaller network. For the network like VANETs which is having high mobility it is not suitable.

\section{BLACKHOLE ATTACK IMPLEMENTATION}

Here discussion is done about the implementation of blackhole attack which is taken with AODV routing protocol for implementation. For managing the routes AODV protocol uses $<$ RREQ,RREP $>$ mechanism. If sender node is not having destination node route details it simple broadcast the RREQ messages. The node within a range will receive the message and reply based on availability of route. This mechanism is having a loophole and attacker can use it to implement blackhole Attack. Fig 2 is presenting RREQ Query packet and Fig. 3 is presenting RREP packet format.

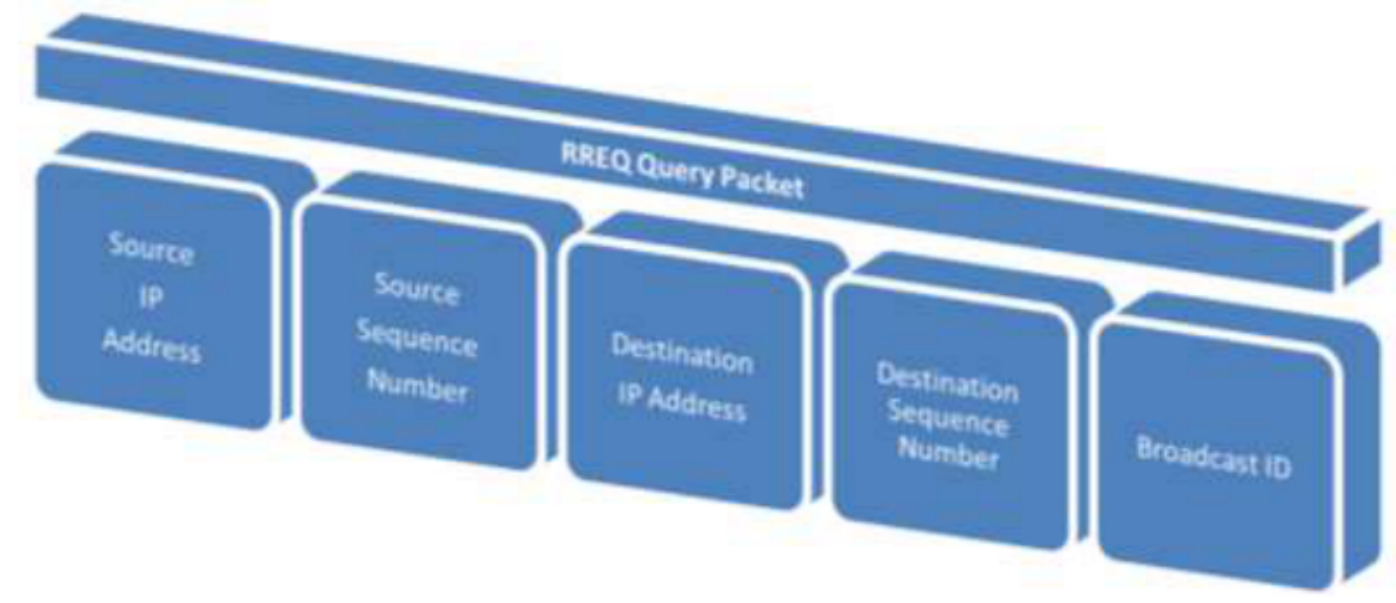

Figure 2 RREQ Query Packet format 


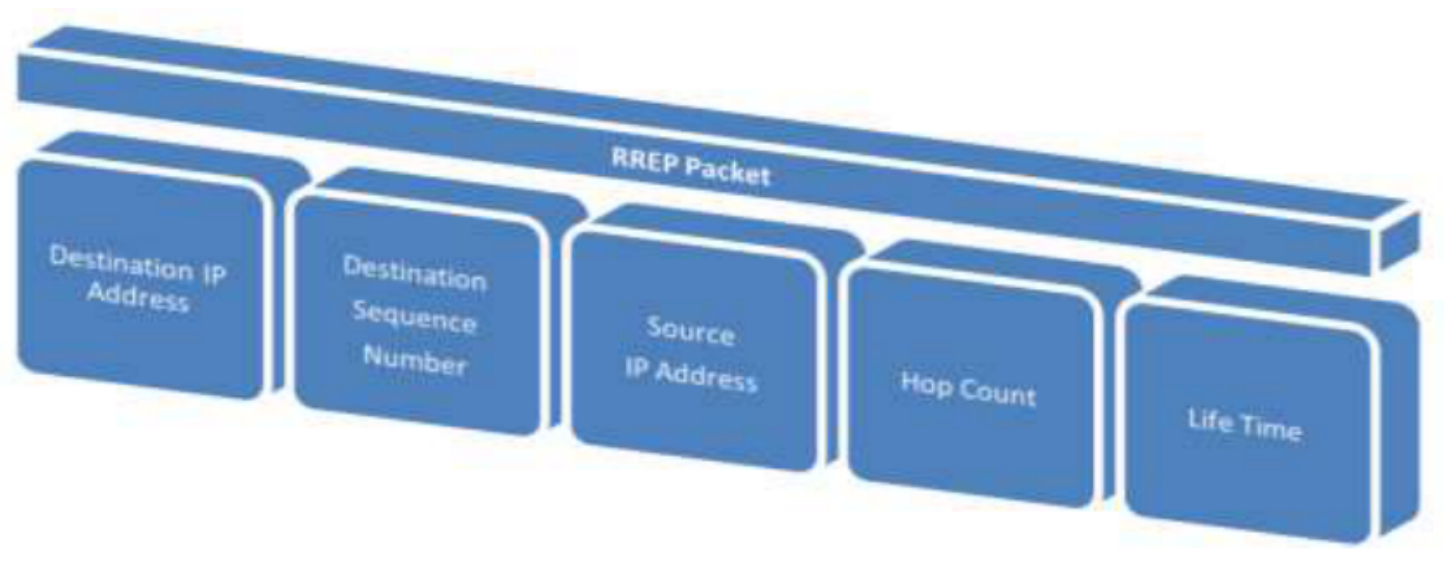

Figure 3 RREP Packet format

Malicious node always in waiting mode for route request and when it receive any RREQ message it immediately reacts and send route reply message (RREP) packet with having best route for the required destination. Size of sequence number is 32 bit and maximum value(threshold value) of that is 4294967295.Malicious node will use this value for sequence number and minimum hop count value (generally it is 1) for attracting traffic.

\section{Algorithm:}

Here blackhole implementation method is discussed using the route reply method. Here notation is taken like SN for Source Node, DN for Destination Node, N_ID for Node ID, MN_ID for Malicious Node ID, SN_SEQ for Source Sequence Number, DN_SEQ for Destination Sequence Number, MAX_SEQ for Maximum Sequence Number, RT for Routing Table, RREQ for Route Request, RREP for Route Reply.

Step 1: Initially source node, which is unaware about the position of destination node broadcast Route Request Message (RREQ).

$$
\mathrm{SN} \rightarrow \text { RREQ [SN_ID, DN_ID, SN_SEQ_NO, BROD_ID] }
$$

Step 2: Different node will receive RREQ broadcasted by Source Node and based on it different node will send Route Reply Message (RREP) to Source Node. Source Node will also get RREP from malicious node.

\section{$\mathrm{SN} \leftarrow$ RREP [DN_ID, SN_ID, DN_SEQ_NO, HOP_COUNT, LIFE_TIME]}

Step 3: Source node will also get reply from malicious node with very high sequence number and having hop count 1 for getting chance in best selection.

$\mathrm{SN} \leftarrow \mathrm{MN}<\operatorname{RREP}\left(\mathrm{SEQ}=\mathrm{MAX} \_\mathrm{SEQ}\right.$, Hop Count=1) $>$

Step 4: Source Node will store all the upcoming RREP messages in Routing Table (RT).

$\mathrm{SN}(\mathrm{RT}) \leftarrow\left\{\mathrm{RREP}\left[\mathrm{DN} \_I D, \mathrm{SN}_{-} \mathrm{ID}, \mathrm{DN} \_\mathrm{SEQ}\right.\right.$ NO, HOP_COUNT, LIFE_TIME] $\}$

Step 5: After getting reply from the different nodes, Source Node (SN) has to choose best route for transmission from the available details of Routing Table (RT). Here the freshness of message will be decided based on Sequence Number. Best route will be chosen on least hop count from fresh entries. So message will forwarded to malicious node.

$\mathrm{MN} \leftarrow \mathrm{SN}<\mathrm{Msg}>$

Step 6: To create the blackhole effect malicious node simple drop the message without forwarding it to the destination. After dropping of data will wait for new RREQ.

$\mathrm{MN}[\mathrm{Drop}<\mathrm{Msg}>$ ] 


\section{BLACKHOLE ATTACK EFFECT IN VANET USING AODV}

Here we considered some of the parameters as a performance Metrics like Packet Drop Rate (PDR), Network Throughput and Average End-to-End Delay. We simulated scenario in NS2 for 100 numbers of nodes for the 1000 second. We have taken the specification as per Table-I for simulating the scenario.

Table 1 Simulation Parameters and Specification

\begin{tabular}{|l|l|}
\hline \multicolumn{1}{|c|}{ Parameters } & \multicolumn{1}{c|}{ Specification } \\
\hline Area Description & Ahmedabad City \\
\hline No. Of Vehicle & 100 \\
\hline Simulation Time & $1000 \mathrm{Sec}$ \\
\hline Type of Vehicle & Car \\
\hline Traffic Light Support & Yes \\
\hline Type of Packet Send & UDP (User Datagram Protocol) \\
\hline Max. Speed of Vehicle & $10 / 20 / 30 \mathrm{~m} / \mathrm{s}$ \\
\hline Length of Vehicle & 3 meter \\
\hline Safe Distance & Front and Rear $-2 \mathrm{~m}$ \\
\hline Allow Overtaking & Yes \\
\hline No. of LAN of Road & 2 \\
\hline Width of LAN & $6 \mathrm{~m}$ \\
\hline Transmission of OBU & $100 \mathrm{~m}$ \\
\hline Transmission of RSU & $250 \mathrm{~m}$ \\
\hline Routing Protocol & AODV \\
\hline Data Packet & 512 bytes \\
\hline Antenna Model & Omni Antenna \\
\hline Simulator & SUMO 0.16.0, MOVE, NS2-2.34 \\
\hline Traffic model & CBR(Constant Bit Rate) \\
\hline Node Movement & Random \\
\hline
\end{tabular}

Here Table-II is representing result of Five observation using AODV Protocol without any attack and Table-III is presenting result of Five observation using AODV Protocol under Blackhole attack. To generate the accurate result, run the same simulation five times with having the same parameters, approaches and methods. By observing the Table-II \& Table-III we found that we received Higher Packet Drop Rate (PDR), End to End delay (E2ED) and Jitter and lower Throughput (Th) and Network Routing Load (NRL). To observe the different variation in result run the simulation five times. After that included malicious nodes inside the topology which creates the effect of blackhole inside the network. Here collaborative blackhole attack is created with the presence of multiple malicious node. Run the simulation again five times under blackhole attack with AODV protocol and presented the result in table III.

Table 2 Simulation Result for AODV Protocol

\begin{tabular}{|c|c|c|c|c|}
\hline & Protocol $\rightarrow$ & & AODV & \\
\hline & No. of Nodes $\downarrow$ & $\begin{array}{c}\text { PDR } \\
(\%)\end{array}$ & $\begin{array}{c}\text { Th } \\
\text { (kbps) }\end{array}$ & $\begin{array}{c}\text { E2ED } \\
(\mathrm{ms})\end{array}$ \\
\hline Observation-1 & \multirow{6}{*}{100} & 3.62 & 117.52 & 71.38 \\
\hline Observation-2 & & 3.53 & 110.58 & 79.04 \\
\hline Observation-3 & & 3.59 & 114.43 & 78.53 \\
\hline Observation-4 & & 3.52 & 115.45 & 70.88 \\
\hline Observation-5 & & 3.57 & 112.88 & 76.06 \\
\hline Average & & 3.57 & 114.17 & 75.18 \\
\hline
\end{tabular}


Dr. Ajay N. Upadhyaya, Dr. J.S. Shah

Table 3 Simulation Result for AODV Protocol Under Blackhole Attack

\begin{tabular}{|c|c|c|c|c|}
\hline & Protocol $\rightarrow$ & \multicolumn{3}{|c|}{ AODV Under Blackhole Attack } \\
\hline & No. of Nodes $\downarrow$ & $\begin{array}{l}\text { PDR } \\
(\%)\end{array}$ & $\begin{array}{c}\text { Th } \\
\text { (kbps) }\end{array}$ & $\begin{array}{c}\text { E2ED } \\
(\mathrm{ms})\end{array}$ \\
\hline Observation-1 & \multirow{6}{*}{100} & 86.63 & 16.30 & 319.04 \\
\hline Observation-2 & & 85.93 & 16.13 & 336.01 \\
\hline Observation-3 & & 86.13 & 16.46 & 338.45 \\
\hline Observation-4 & & 86.29 & 16.41 & 309.27 \\
\hline Observation-5 & & 85.91 & 16.49 & 322.73 \\
\hline Average & & 86.18 & 16.36 & 325.10 \\
\hline
\end{tabular}

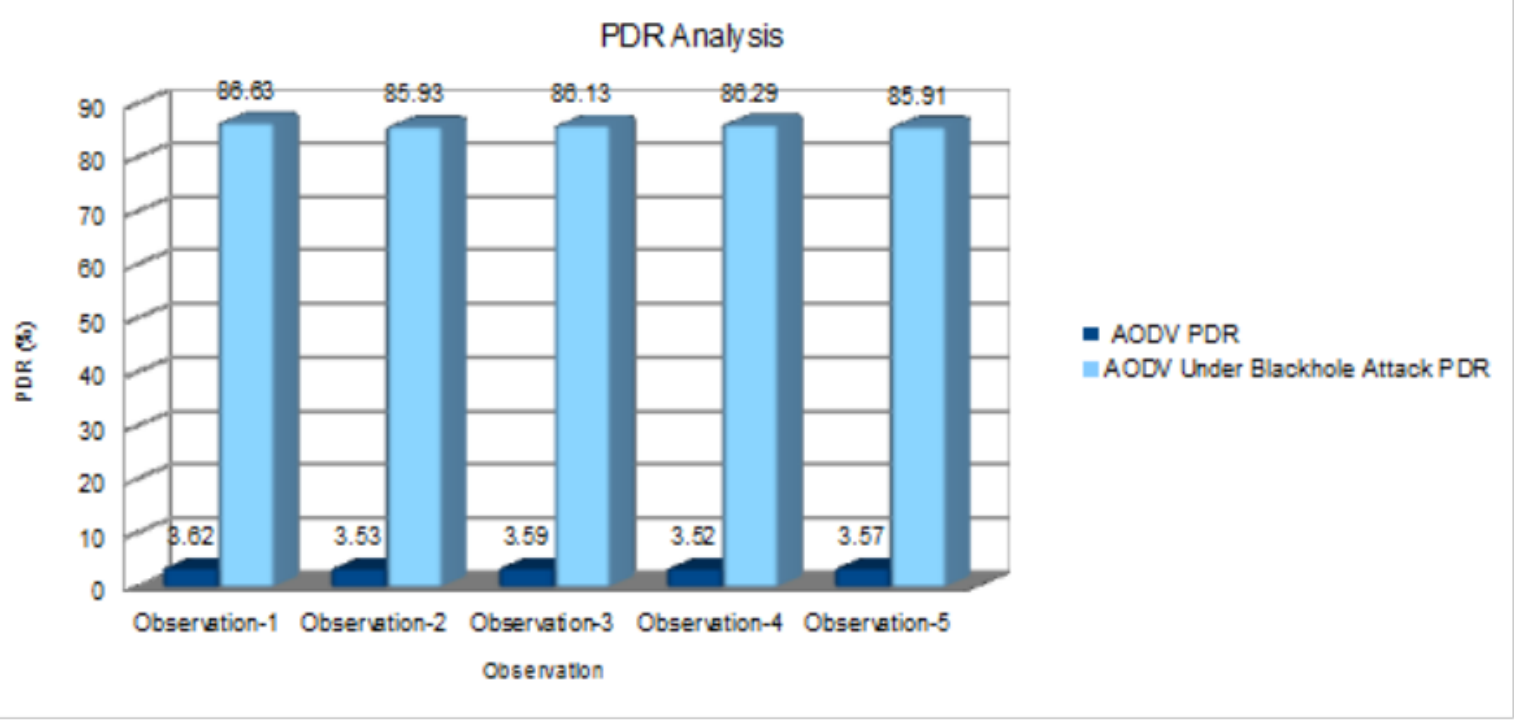

Figure 4 PDR Analysis

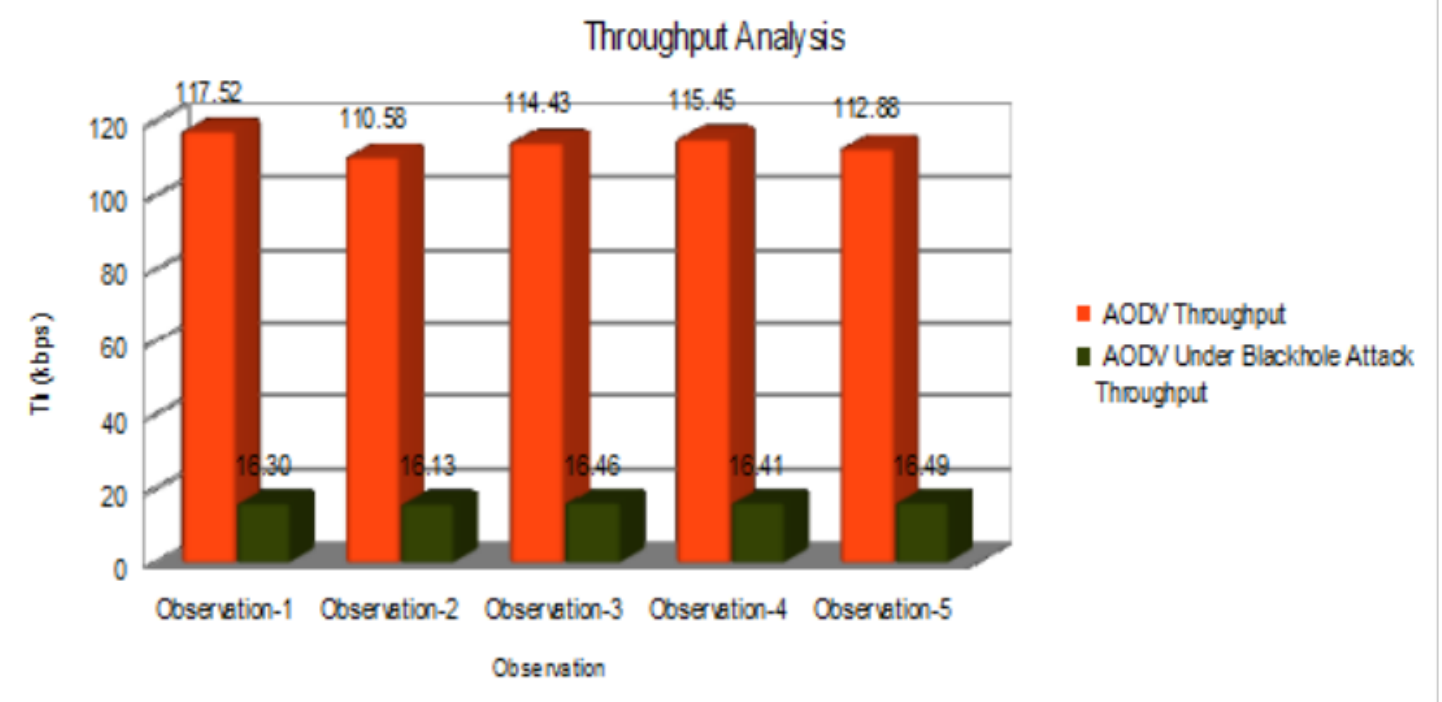

Figure 5 Throughput Analysis 


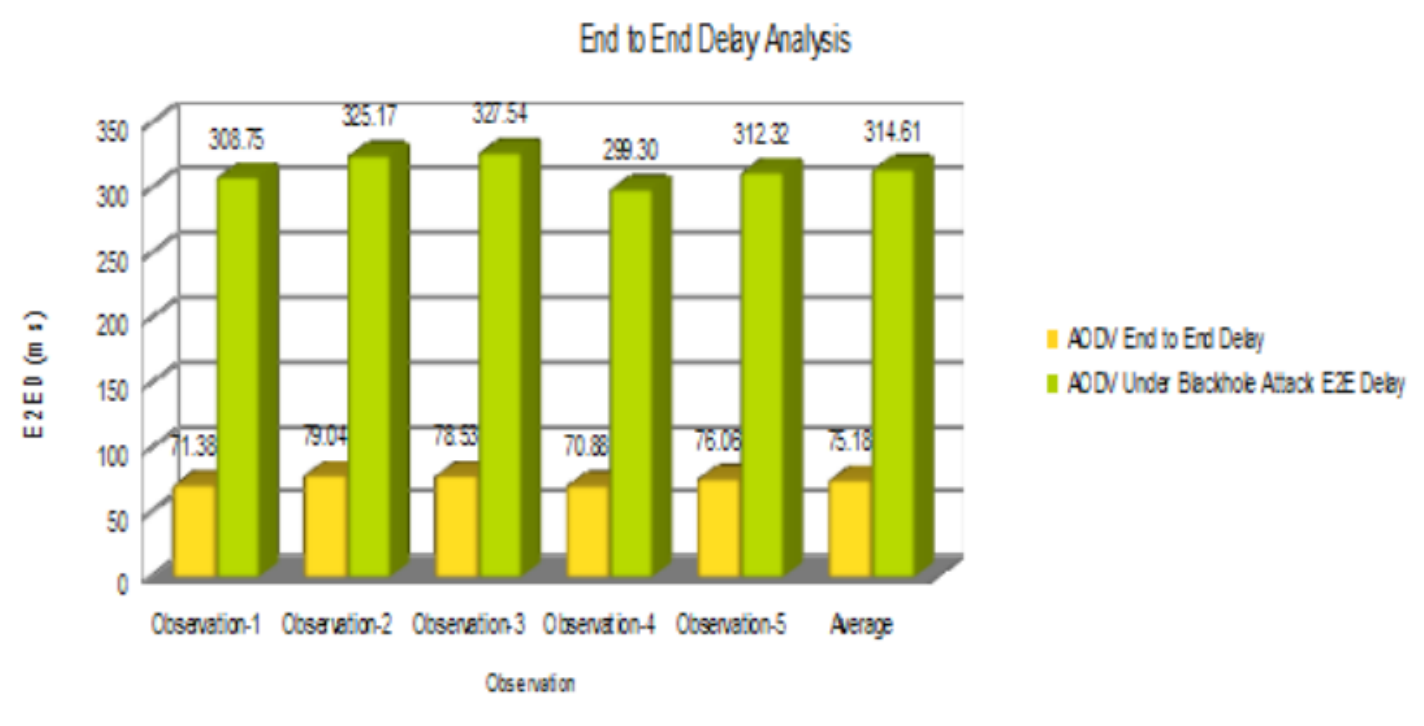

Figure 6 End to End Delay Analysis

Figure 4 is presenting Packet Drop Rate Analysis, Figure 5 is presenting Throughput Analysis and Figure 6 is presenting Average End to End Delay Analysis for AODV Protocol behaviour and AODV Protocol behaviour under Blackhole Attack. First run the simulation as simple case by using AODV protocol without any effect of blackhole attack and received the average packet drop rate $3.57 \%$ for 100 vehicles and After that run same scenario under collaborative Blackhole Attack and received the Packet Loss $86.18 \%$ for 100 vehicles. After analyzing Packet Drop Rate analyzed Throughput parameter of simple case by using AODV protocol without any effect of blackhole attack and received the Throughput rate $114.17 \mathrm{kbps}$ for 100 vehicles and After that run same scenario under collaborative Blackhole Attack and received Throughput $16.36 \mathrm{kbps}$ for 100 vehicles. After analyzing Packet Drop Rate \& Throughput, analyzed Average End to End Delay parameter of simple case by using AODV protocol without any effect of blackhole attack and received the Average End to End Delay $75.18 \mathrm{~ms}$ for 100 vehicles and After that run same scenario under collaborative Blackhole Attack and received Average End to End Delay 325.10 ms for 100 vehicles.

\section{CONCLUSIONS}

Reactive routing protocol is more preferable for VANET application but on demand routing protocol DSR is not suitable for the large network so AODV Routing protocol is chosen. AODV is also having some limitation and it is most vulnerable protocol under the routing attacks like Blackhole. But there is a more scope of improvement in AODV which we selected for our work. In this paper we presented Blackhole Routing Attack under AODV Protocol and presented comparative analysis of with and without Blackhole attack effect. We found higher Packet Drop Rate and Average End to End Delay and lower Throughput under Blackhole Attack. We presented here Effect of Blackhole attack in VANET and in future we will work on different Blackhole Attack detection and prevention methods. 


\section{REFERENCES}

[1] J. Nzouonta, N. Rajgure, G. Wang and C. Borcea, "VANET Routing on City Roads Using Real-Time Vehicular Traffic Information," in IEEE Transactions on Vehicular Technology, vol. 58, no. 7, pp.,2008, 3609-3626.

[2] F. Li and Y. Wang, "Routing in vehicular ad hoc networks: A survey," in IEEE Vehicular Technology Magazine, vol. 2, no. 2, pp. 12-22. 2007.

[3] S. Sarika, A. Pravin, A. Vijayakumar, K. Selvamani, Security Issues in Mobile Ad Hoc Networks, Procedia Computer Science, Volume 92, 2016, Pages 329-335, ISSN 18770509 .

[4] Navjot Kaur, Sandeep Kad, A Review on Security Related Aspects in Vehicular Adhoc Networks, Procedia Computer Science, Volume 78, 2016, Pages 387-394, ISSN 18770509 .

[5] Bharati Mishra, Priyadarshini Nayak, Subhashree Behera, and Debasish Jena. 2011. Security in vehicular adhoc networks: a survey. In Proceedings of the 2011 International Conference on Communication, Computing \& Security (ICCCS '11). ACM, New York, NY, USA, 590-595.

[6] G. Samara, W. A. H. Al-Salihy and R. Sures, "Security issues and challenges of Vehicular Ad Hoc Networks (VANET)," 4th International Conference on New Trends in Information Science and Service Science, Gyeongju, 2010, pp. 393-398.

[7] J. Zuo, Y. Wang, Y. Liu and Y. Zhang, "Performance Evaluation of Routing Protocol in VANET with Vehicle-Node Density," 2010 IEEE 6th International Conference on Wireless Communications Networking and Mobile Computing (WiCOM), Chengdu, 2010, pp. 1-4.

[8] A. K. Basil, M. Ismail, M. A. Altahrawi, H. Mahdi and N. Ramli, "Performance of AODV and OLSR routing protocols in VANET under various traffic scenarios," 2017 IEEE 13th Malaysia International Conference on Communications (MICC), Johor Bahru, 2017, pp. 107-112

[9] M. Jain and R. Saxena, "Overview of VANET: Requirements and its routing protocols," 2017 IEEE International Conference on Communication and Signal Processing (ICCSP), Chennai, 2017, pp. 1957-1961.

[10] D. Sutariya and S. Pradhan, "An improved AODV routing protocol for VANETs in city scenarios," IEEE-International Conference On Advances In Engineering, Science And Management (ICAESM -2012), Nagapattinam, Tamil Nadu, 2012, pp. 575-581. 